\title{
STELLA Experiment: Design and Model Predictions
}

W.D. Kimura, M. Babzien, I. Ben-Zvi, L.P. Campbell, D.B. Cline,

R.B. Fiorito, J.C. Gallardo, S.C. Gottschalk, P. He, K.P. Kusche,

Y. Liu, R.H. Pantell, I.V. Pogorelsky, D.C. Quimby,

K.E. Robinson, D.W. Rule, J. Sandweiss, J. Skaritka, 0 C IVED

A. van Steenbergen, L.C. Steinhauer and V. Yakimenko

STI Optronics, Inc., Bellevue, WA 98004

Brookhaven National Laboratory, Upton, NY 11973

APR 051939

UCLA, Los Angeles, CA 90024

Catholic University of America, Washington, DC 20064

Stanford University, Stanford, CA 94305

Naval Surface Warfare Center, West Bethesda, MD 20817

Yale University, New Haven, CT 06520

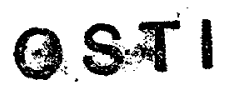

July 1998

National Synchrotron Light Source

Brookhaven National Laboratory

Operated by

Brookhaven Science Associates

Upton, NY 11973 


\section{DISCLAIMER}

This report was prepared as an account of work sponsored by an agency of the United States Government. Neither the United States Government nor any agency thereof, nor any of their employees, nor any of their contractors, subcontractors or their employees, makes any warranty, express or implied, or assumes any legal liability or responsibility for the accuracy, completeness, or any third party's use or the results of such use of any information, apparatus, product, or process disclosed, or represents that its use would not infringe privately owned rights. Reference herein to any specific commercial product, process, or service by trade name, trademark, manufacturer, or otherwise, does not necessary constitute or imply its endorsement, recommendation, or favoring by the United States Government or any agency thereof or its contractors or subcontractors. The views and opinions of authors expresses herein do not necessarily state to reflect those of the United States Government or any agency thereof. 


\section{DISCLAIMER}

Portions of this document may be illegible in electronic image products. Images are produced from the best available original document. 


\title{
STELLA Experiment: Design and Model Predictions
}

\author{
W. D. Kimura, ${ }^{*}$ M. Babzien, ${ }^{\dagger}$ I. Ben-Zvi, ${ }^{\dagger}$ L. P. Campbell, ${ }^{*}$ D. B. Cline ${ }^{\ddagger}$ \\ R. B. Fiorito,${ }^{\S}$ J. C. Gallardo,${ }^{\dagger}$ S. C. Gottschalk, ${ }^{*}$ P. He ${ }^{\ddagger}$ K. P. Kusche, ${ }^{*}{ }^{\dagger}$ \\ Y. Liu, ${ }^{\ddagger}$ R. H. Pantell, ${ }^{\ddagger}$ I. V. Pogorelsky, ${ }^{\dagger}$ D. C. Quimby, ${ }^{*}$ \\ K. E. Robinson, ${ }^{*}$ D. W. Rule, ${ }^{\sharp}$ J. Sandweiss, ${ }^{\forall}$ J. Skaritka, ${ }^{\dagger}$ \\ A. van Steenbergen, ${ }^{\dagger}$ L.C. Steinhauer, ${ }^{*}$ and V. Yakimenko ${ }^{\dagger}$
}

\author{
*STI Optronics, Inc., Bellevue, WA 98004 \\ ${ }^{\dagger}$ Brookhaven National Laboratory, Upton, NY 11973 \\ ${ }^{\ddagger}$ UCLA, Los Angeles, CA 90024 \\ ${ }^{8}$ Catholic University of America, Washington DC 20064 \\ Stanford University, Stanford, CA 94305 \\ \# Naval Surface Warfare Center, West Bethesda, MD 20817 \\ ${ }^{\forall}$ Yale University, New Haven, CT 06520
}

\begin{abstract}
The STaged ELectron Laser Acceleration (STELLA) experiment will be one of the first to examine the critical issue of staging the laser acceleration process. The BNL inverse free electron laser (IFEL) will serve as a prebuncher to generate $\sim 1-\mu \mathrm{m}$ long microbunches. These microbunches will be accelerated by an inverse Cerenkov acceleration (ICA) stage. A comprehensive model of the STELLA experiment is described. This model includes the IFEL prebunching, drift and focusing of the microbunches into the ICA stage, and their subsequent acceleration. The model predictions will be presented, including the results of a system error study to determine the sensitivity to uncertainties in various system parameters.
\end{abstract}

\section{INTRODUCTION}

Exciting progress has been made in the last several years on laser accelerators including energy gains of $100 \mathrm{MeV}$ and $\mathrm{GeV} / \mathrm{m}$ acceleration gradients (1). At the BNL Accelerator Test Facility (ATF), there are two laser acceleration experiments - an inverse Cerenkov accelerator (ICA) (2) and an inverse free electron laser (IFEL) (3). Both of these experiments have been routinely accelerating electrons. Thus, laser acceleration is becoming more viable as an advanced acceleration technique. 
Now the next logical step in its evolution is to address the issue of multiaccelerator module staging and acceleration of the microbunches produced during the laser acceleration process. To efficiently accelerate the electrons throughout these stages it is necessary to prebunch the electrons into a microbunch whose longitudinal length is a small fraction of the accelerating wave. In laser accelerators this accelerating wave can be of order $10 \mu \mathrm{m}$. Evidence of microbunching at optical wavelengths has already been detected from the IFEL using a coherent transition radiation (CTR) diagnostic (4).

Staging requires rephasing the microbunches with the accelerating light wave. Efficient acceleration of the electrons contained within a microbunch also requires trapping the electrons within the acceleration potential well. This implies the need to minimize effects that can lead to bunch smearing where the electrons no longer stay within the main bunch distribution. This may be due to trajectory differences of the electrons within the microbunch, gas scattering effects in the case of ICA, and spacecharge spreading. While these effects are analogous to those encountered in microwave linacs, they can have a much greater impact in laser acceleration because of the orders of magnitude shorter wavelengths that are involved.

Therefore, the primary goal of the Staged Electron Laser Acceleration (STELLA) experiment is to demonstrate effective trapping and acceleration within an ICA acceleration stage of microbunches generated by an IFEL. During the course of this experiment, we will be addressing the many issues related to generation and preservation of the microbunch, and effective control of the rephasing process. These results will directly benefit other laser acceleration research by demonstrating that efficient staging is possible. This accomplishment will open the door to the next step of adding multiple laser acceleration stages in series to achieve high net energy gain.

\section{DESCRIPTION OF EXPERIMENT}

Figure 1 is a conceptual layout of the STELLA experiment. The $e$-beam from the ATF linac, which consists of a single 10-ps macropulse, enters the IFEL prebuncher from the left. A beam splitter sends a relatively small amount of laser power to the IFEL for modulating the electron energy. The objective is to modulate the energy just enough so that maximum bunching occurs at the end of the drift region just before the entrance to the ICA laser acceleration stage. The rest of the laser power passes through a trombone delay line and to the ICA stage. This trombone delay line will be used to adjust the relative phase of the laser light in the ICA cell with the microbunches created by the IFEL.

For a given wiggler configuration (i.e., spacing and magnetic field strength), $e$ beam energy, and laser wavelength, the optimum bunching distance for the IFEL is controlled by the amount of energy modulation imparted by the laser beam. Therefore, 


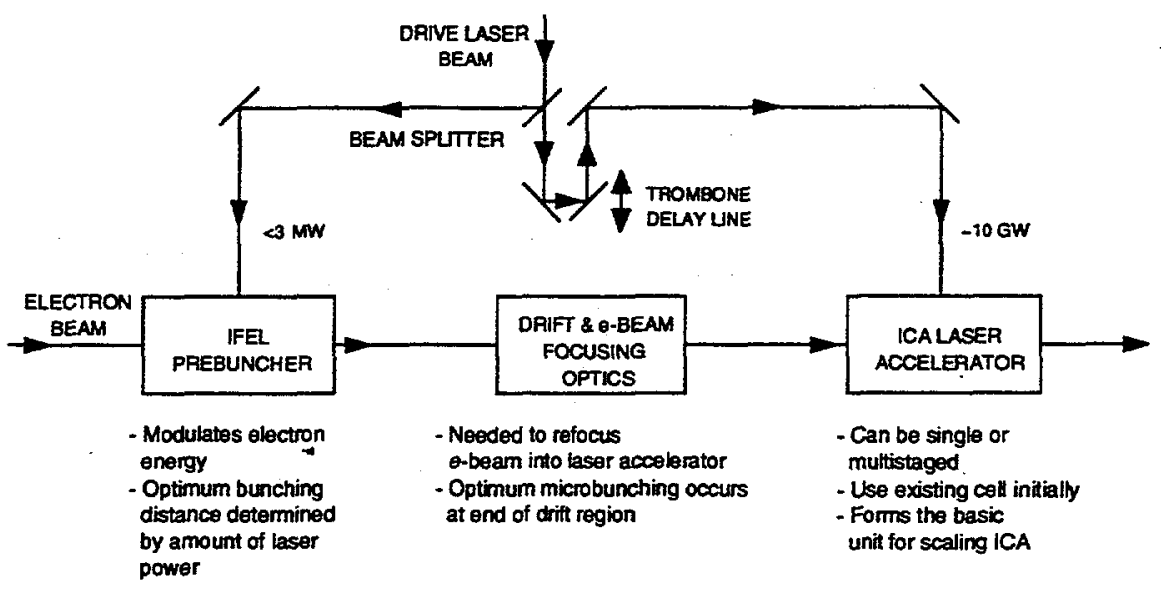

FIGURE 1. Conceptual layout for STELLA experiment.

during the experiment the IFEL prebunching characteristics can be "tuned" by adjusting the amount of laser power sent into the IFEL.

Between the IFEL and the ICA gas cell is a drift space in which quadrupoles will be located to focus the $e$-beam into the ICA gas cell. Next comes the ICA laser accelerator stage located at the end of the drift distance. The primary technical issue here is properly rephasing the optically bunched beam with the laser light wave in the ICA cell so that the bunch is trapped and accelerated.

\section{IFEL, ICA, and ATF System Parameters for STELLA}

A schematic layout of the BNL IFEL (3) is given in Fig. 2. The ATF $\mathrm{CO}_{2}$ laser beam is focused into a $2.8 \mathrm{~mm} \mathrm{ID} 60-\mathrm{cm}$ long sapphire circular waveguide located inside the $47-\mathrm{cm}$ long wiggler. The guide extends beyond the front of the wiggler to permit the proper laser mode $\left(\mathrm{HE}_{11}\right)$ to form within the guide and eliminate other unwanted modes.

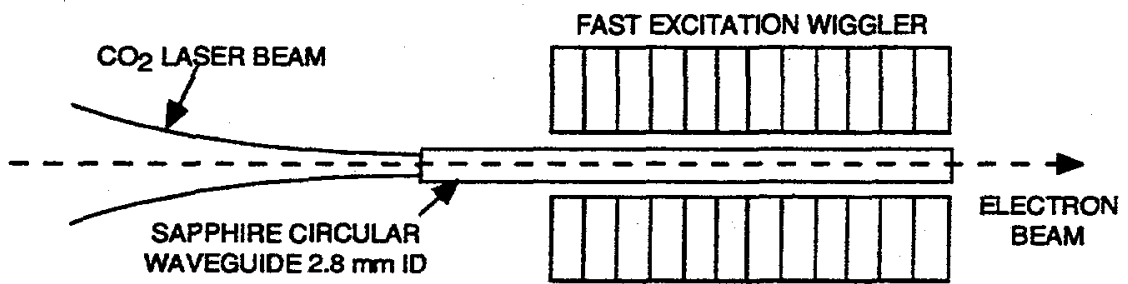

FIGURE 2. Schematic layout for BNL IFEL experiment. 


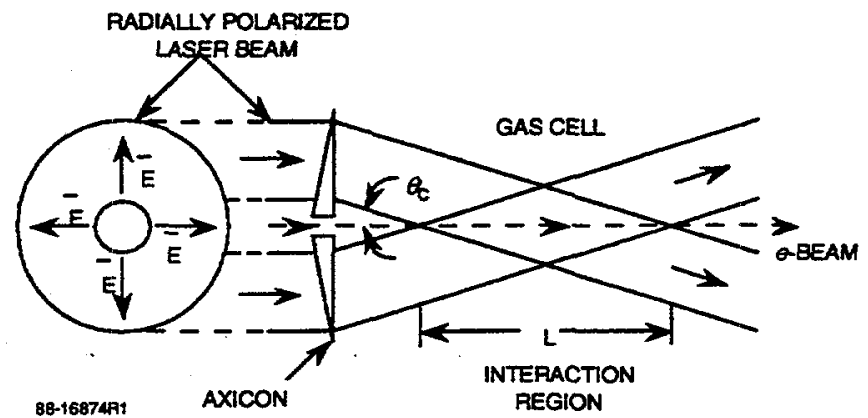

FIGURE 3. Basic geometry for inverse Cerenkov accelerator. From Ref. (6).

From earlier analysis (5) the IFEL wiggler parameters for STELLA have been selected to be: wiggler length $L_{\mathrm{w}}=47 \mathrm{~cm}, \lambda_{\mathrm{w}}=3.3 \mathrm{~cm}$ (untapered), gap $=4 \mathrm{~mm}, K$ parameter $=2.86$, and on-axis peak field $=0.93 \mathrm{~T}$. An untapered wiggler was found to provide the needed amount of energy modulation while at the same time being more tolerant of variations in the $e$-beam and laser beam parameters.

Figure 3 shows the basic scheme for the inverse Cerenkov accelerator. A radially polarized laser beam is focused by an axicon onto the $e$-beam traveling through a gas cell filled with $\mathrm{H}_{2}$ gas (6). The laser light intersects the $e$-beam at the Cerenkov angle for phase matching, i.e., $\theta_{c}=\cos ^{-1}(1 / n \beta)$, where $n$ is the index of refraction of the gas, and $\beta$ is the ratio of electron velocity to the velocity of light. The electrons enter and exit the gas cell through thin diamond windows.

Previous analysis (5) demonstrated the importance of minimizing electron scattering off the windows and gas molecules. Scattering degrades the ability to trap the electrons within the microbunch. During STELLA the gas cell is being modified to reduce scattering by using thinner diamond windows ( $1 \mu \mathrm{m}$ thick instead of $2.1 \mu \mathrm{m}$ ) and shortening the interaction length to $6.5 \mathrm{~cm}$. With these changes, the model predicts that the effects of scattering will be reduced to the point where other effects, such as emittance and intrinsic energy spread, become dominant factors. The other gas cell parameters remain the same, i.e., $\theta_{c}=20 \mathrm{mrad}$ and gas pressure $\approx 1.8 \mathrm{~atm}$.

Table 1 lists the system requirements for the ATF linac and ATF psec $\mathrm{CO}_{2}$ laser. These were determined from the integrated modeling analysis discussed below. We should note that most of these values have been already demonstrated by the ATF or are achievable with the present system.

\section{DESCRIPTION OF DESIGN ANALYSIS}

The design of the STELLA experiment is made more complex by the need to create and maintain micron-sized microbunches throughout the system. To understand how the various physical parameters affect this process, an integrated model was 
TABLE 1. ATF System Requirements for STELLA.

\begin{tabular}{lc}
\hline Parameter & Value Needed for STELLA \\
\hline Electron Beam & $45 \mathrm{MeV}$ \\
$\quad$ Mean energy, $E$ & $\leq 0.85 \pi \mathrm{mm}$-mrad \\
$\quad$ Normalized emittance, $\varepsilon$ & $0.15 \% \mathrm{rms}$ FWHM \\
Intrinsic energy spread, $\Delta E$ & $\geq 0.1 \mathrm{nC}$ \\
$\quad$ Macropulse charge, $Q$ & $10.6 \mu \mathrm{m}$ \\
Laser Beam & $2.6 \mathrm{MW}$ (linearly polarized) \\
$\quad$ Laser wavelength & $\geq 10 \mathrm{GW}$ (radially polarized) \\
$\quad$ Power delivered to IFEL & \\
$\quad$ Power delivered to ICA &
\end{tabular}

developed, which consists of several models combined together to simulate the entire STELLA experiment. It uses a 1-D FEL tracking code developed at STI Optronics, Inc. (STT) for the IFEL wiggler. A 3-D ray tracing code was written to track the electrons through the drift region between the IFEL and ICA gas cell. Finally, the STI Monte Carlo ICA model (7), which includes the effects of scattering, was used to predict the acceleration of the microbunches. The integrated model includes all important effects, except space charge. Separate analysis (8) of space charge effects indicate that for the anticipated STELLA conditions it will be at most a $10 \%$ effect.

The 1-D IFEL model tracks the electron dynamics in $(\gamma, \psi)$ phase space. It assumes that the $e$-beam is matched and aligned within the wiggler, the optical field is uniform along the wiggler length, the optical pulse is long ( $-200 \mathrm{ps})$ compared to the electron pulse ( $-10 \mathrm{ps}$ ), and the energy spread distribution is Gaussian. Emittance effects within the wiggler have been neglected, which is reasonable since the $e$-beam diameter is much smaller than the optical beam.

The drift region model includes the capability to simulate bunch smearing. TRANSPORT is used to design the $e$-beam optics (i.e., triplet between the IFEL and ICA systems). Differences in the axial velocity, path length difference, and optical phase relative to the resonant on-axis electron are calculated for each Monte Carlo electron traveling from the IFEL through the drift region. Chromatic effects are included by accounting for the magnetic rigidity of individual particles. Misalignments are simulated by introducing mis-steering at the wiggler exit and applying correction kicks at the triplet exit and ICA cell entrance.

The ICA model has been used extensively in the past to simulate the ICA process. It incorporates all relevant physics except space charge effects, including axicon focusing of the laser beam, optical beam propagation with diffraction and interference effects of an ideal laser beam, Rutherford scattering caused by the gas and $e$-beam windows, straggling and other inelastic losses, and the full $e$-beam characteristics (e.g., emittance, energy spread, etc.). A new version of the model has recently been developed that can handle nonideal laser beams, e.g., nonaxisymmetric, nonuniform, and imperfect radial polarization. It has not been applied to STELLA yet. 


\section{SENSITIVITY ANALYSIS RESULTS}

The integrated model was run to understand how variations in the different system parameters affect the experiment. The objective is to trap and accelerate the microbunch within a relatively narrow energy range (goal: $<5 \%$ ) while maintaining the original microbunch length (goal: $<1 \mu \mathrm{m}$ ). During the first phase of the experiment there will be less emphasis on demonstrating high amounts of acceleration; although, with $10 \mathrm{GW}$ delivered to the accelerator, an energy gain of nearly $10 \mathrm{MeV}$ is predicted, corresponding to an acceleration gradient of $>150 \mathrm{MeV} / \mathrm{m}$.

For the baseline conditions, the model predicts for $2.6 \mathrm{MW}$ delivered to the IFEL prebuncher a sinusoidal energy modulation of $\approx \pm 0.55 \%( \pm 0.25 \mathrm{MeV})$. At the end of the drift region just before the ICA cell, roughly $50 \%$ of the electrons are bunched together with a microbunch length of $\approx 0.84 \mu \mathrm{m}$ FWHM.

There are various effects that can degrade the prebuncher. One major effect is the intrinsic energy spread of the incoming $e$-beam, which directly affects the bunch length as depicted in Fig. 4. A baseline value of $0.15 \%$ has been chosen for the experiment.

$E$-beam energy stability affects the phase jitter of the microbunches leaving the IFEL, which can cause problems later in the accelerator because of phase mismatch with the optical field in the ICA cell. The model predicts a detuning of 2.7 radians per $\%$ of energy detuning. This implies the need for an energy jitter of $<+0.11 \%$.

Low emittance appears to be less critical for the prebuncher than the accelerator (see below). Emittance degrades the wiggler interaction by reducing the overlap with the laser beam. It also leads to path length differences in the drift region causing bunch smearing.

The prebuncher is also less sensitive to laser power fluctuations. This is demonstrated in Fig. 5. Fluctuation of $\pm 50 \%$ are tolerable.

Table 2 summarizes the major sensitivities of the prebuncher.

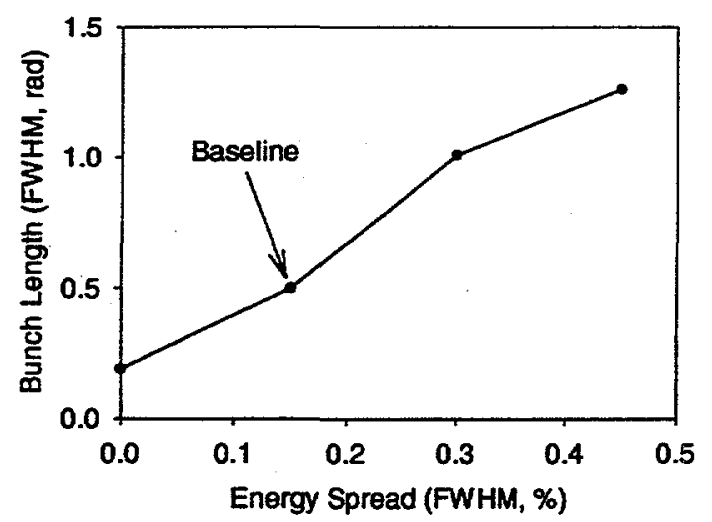

FIGURE 4. Scaling of bunch length with $e$-beam energy spread. 


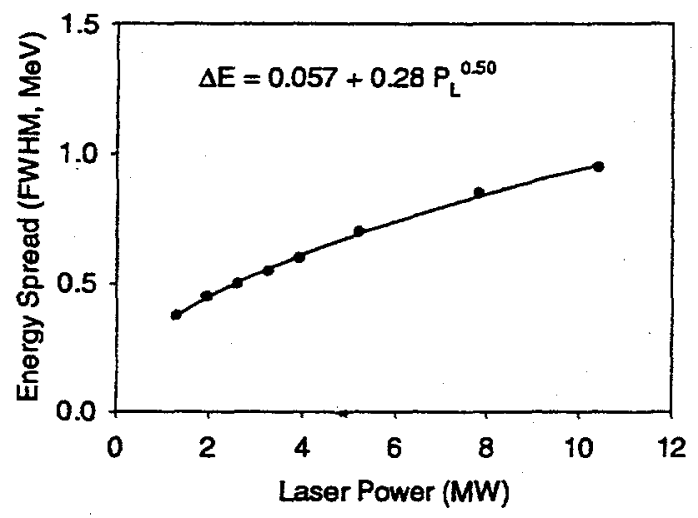

(a)

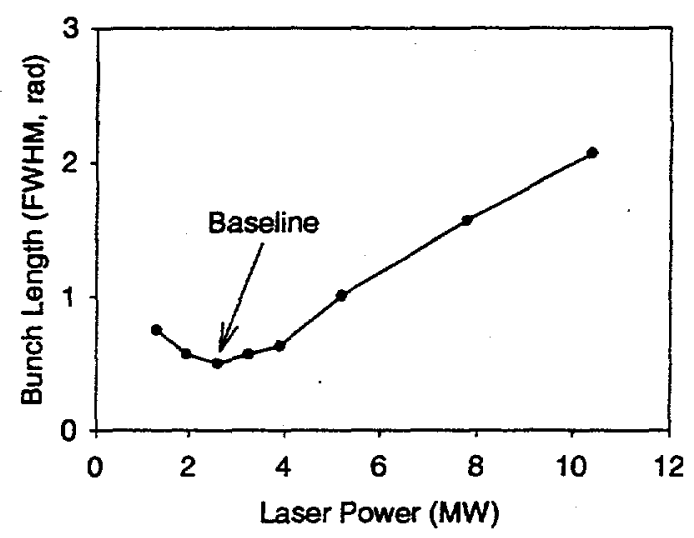

(b)

FIGURE 5. Scaling of induced energy spread and bunch length with laser power delivered to IFEL (a) Laser-induced energy versus laser power. (b) Bunch length versus laser power.

The baseline predictions for the output of the ICA accelerator are given in Fig. 6 . This shows a net energy gain of $\approx 10 \mathrm{MeV}$ and bunch length of $<1 \mu \mathrm{m}$. (The second small peak in Fig. 6(b) is a remnant from the initial prebuncher energy modulation.)

$E$-beam emittance strongly affects the ICA acceleration process. This is shown in Fig. 8. Although the peak energy gain does not change with emittance very much, the width of the energy peak and the bunch length tend to broaden with larger emittance.

In the ICA accelerator, small $e$-beam size and accurate alignment through the gas cell is critical. This is demonstrated in Fig. 8. Note, the laser beam size in the axicon focal region is $\approx 200 \mu \mathrm{m}$ FWHM. Hence, it is important that the $e$-beam diameter along the axicon focal region remain a fraction of this laser beam size in order for all the electrons within the microbunch to experience similar amounts of acceleration. Our goal is to have an $e$-beam diameter of $\approx 60 \mu \mathrm{m}$.

TABLE 2. Summary of Major Prebuncher Sensitivities.

\begin{tabular}{lll}
\hline Parameter & \multicolumn{1}{c}{ Tolerance } & \multicolumn{1}{c}{ Deleterious Effect } \\
\hline $\begin{array}{l}\text { Initial energy spread variance } \\
\text { from baseline } \\
E \text {-beam energy jitter }\end{array}$ & $\Delta E / E<0.3 \% \mathrm{FWHM}$ & Bunch smearing \\
& $\Delta E< \pm 0.11 \%$ & $\begin{array}{l}\text { Causes bunch to appear at } \\
\text { variable phase. Weakly affects } \\
\text { bunching. }\end{array}$ \\
Emittance & $\begin{array}{l}\text { Bunch smearing in drift region. } \\
\text { May also reduce IFEL bunching } \\
\end{array}$ & $\begin{array}{l}\text { efficiency (not included yet in } \\
\text { IFEL model). }\end{array}$ \\
\hline
\end{tabular}




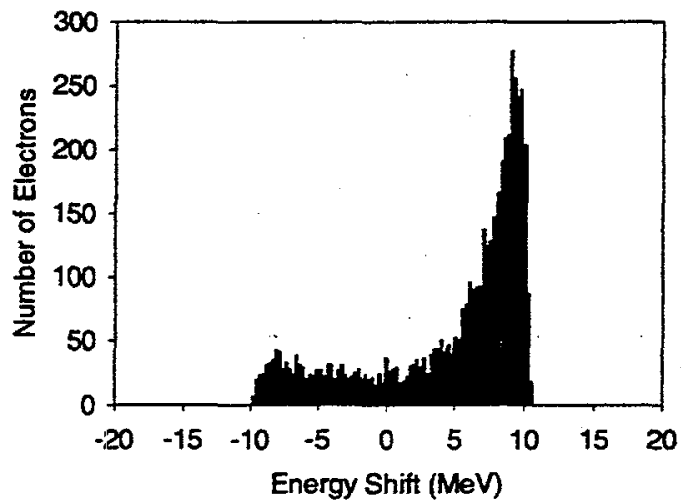

(a)

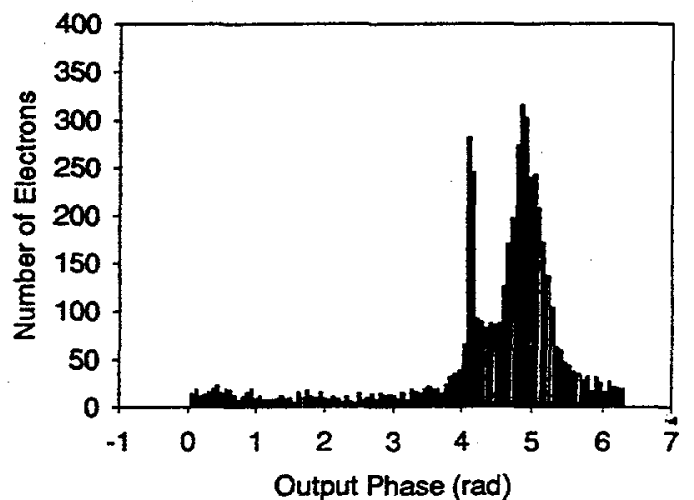

(b)

FIGURE 6. Output of ICA accelerator for $E=45 \mathrm{MeV}, \varepsilon_{\mathrm{n}}=0.5 \pi \mathrm{mm}-\mathrm{mrad} \mathrm{rms}, \Delta E / E=0.15 \%$

FWHM, 1- $\mu \mathrm{m}$ windows, with gas scattering. (a) Energy spectrum. (b) Longitudinal density.

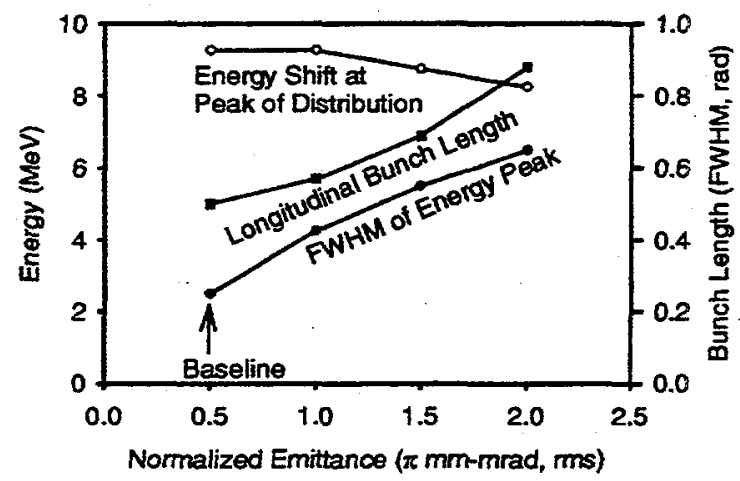

FIGURE 7. Scaling of ICA acceleration parameters with e-beam emittance.

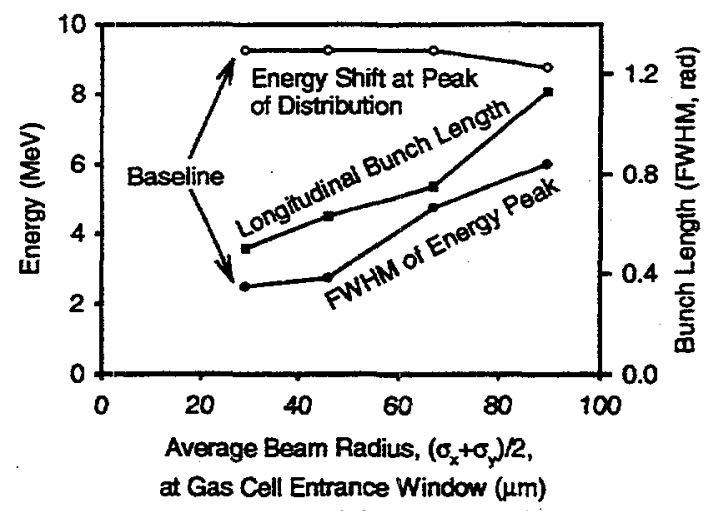

(a)

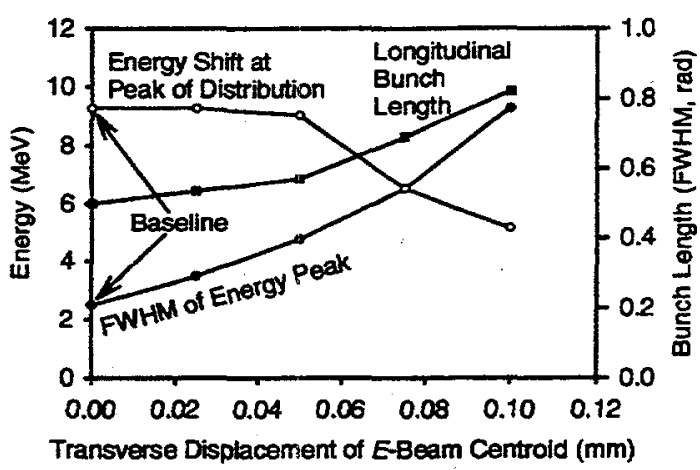

(b)

FIGURE 8. Scaling of ICA acceleration parameters with (a) $e$-beam size and (b) displacement. 


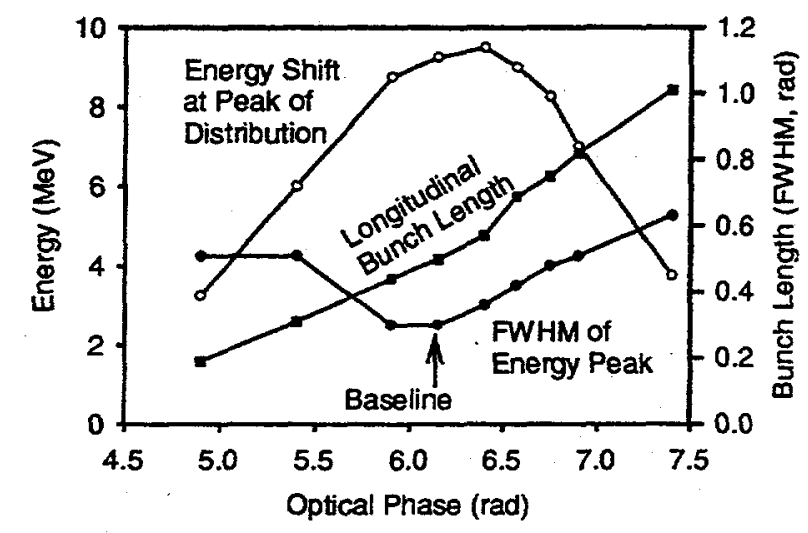

FIGURE 9. Variation of ICA acceleration parameters due to phase variation between electron microbunch and laser beam.

Not surprising, it is important to maintain tight control of the microbunch phase relative to the optical field inside the gas cell. Shifts in phase can be caused by fluctuations in the $e$-beam energy and the laser beam path length. Figure 9 shows the sensitivity to phase jitter. Note, in our simulations maximum acceleration and minimum energy spread of the accelerated bunch occurs at a phase value of 6.15 radians.

Based upon the simulations we have established an overall phase jitter tolerance of $\pm 0.40 \mathrm{rad}( \pm 0.64 \mu \mathrm{m}$ total path difference). This corresponds to an acceptable laser beam path length jitter of $\approx \pm 0.45 \mu \mathrm{m}$ and $e$-beam energy jitter of $\approx \pm 0.11 \%$, assuming these effects add in quadrature because they are uncorrelated.

The ICA process is more tolerant of laser power fluctuations with a tolerance of $\pm 32 \%$ being chosen for STELLA.

Table 3 summarizes the major sensitivities of the ICA accelerator.

TABLE 3. Summary of Major ICA Accelerator Sensitivities.

\begin{tabular}{lll}
\hline Parameter & \multicolumn{1}{c}{ Tolerance } & \multicolumn{1}{c}{ Deleterious Effect } \\
\hline Emittance & $\begin{array}{l}\varepsilon_{\mathrm{n}}<0.85 \pi \mathrm{mm} \text {-mrad rms } \\
\text { (Note, more stringent than IFEL } \\
\text { requirement.) } \\
\Delta E< \pm 0.11 \%\end{array}$ & $\begin{array}{l}\text { Bunch smearing and loss of } \\
\text { overlap with laser beam causes } \\
\text { energy smearing. }\end{array}$ \\
$\begin{array}{l}E \text {-beam energy jitter } \\
\begin{array}{l}\text { Mis-steering of } e \text {-beam through } \\
\text { gas cell }\end{array}\end{array}$ & $\Delta r \leq 25 \mu \mathrm{m}$ & $\begin{array}{l}\text { Causes jitter of initial bunch } \\
\text { phasing. }\end{array}$ \\
$\begin{array}{l}\text { Phase jitter } \\
\text { Reduces overlap with laser }\end{array}$ & $\begin{array}{l}\text { Reduch } \\
\text { beam. } \\
\text { Reduces bunch acceleration and } \\
\text { increases bunch energy spread. }\end{array}$ \\
\hline
\end{tabular}




\section{CONCLUSION}

An integrated model of the STELLA experiment has been developed, which predicts the sensitivity to variations in system parameters. This has identified key experimental issues, which are being addressed in the hardware design of the experiment (9). The experiment is currently being assembled and tested. Initial measurements will be conducted using $10 \mathrm{GW}$ laser power to the ICA cell. Plans are to later modified the system to handle much higher laser power (e.g., several hundred gigawatts).

Additional information on the STELLA experiment can be found on the NSLS ATF Web Site at: http://www.nsls.bnl.gov/AccTest/experiments/STELLA/STELLA.htm.

\section{ACKNOWLEDGMENTS}

The authors wish to acknowledge Dr. J. R. Fontana for his technical advice during the initial planning of this experiment; and Dr. Xijie Wang for his on-going assistance and support. This work was supported by the U.S. Department of Energy, Grant Nos. DE-FG03-98ER41061, DE-AC02-76CH00016, and DE-FG03-92ER40695.

\section{REFERENCES}

1. See for example Proceedings of Workshop on Advanced Acceleration Concepts, Oct. 12-18, 1996, Lake Tahoe, CA.

2. Kimura, W. D., Kim, G. H., Romea, R. D., Steinhauer, L. C., Pogorelsky, I. V., Kusche, K. P., Fernow, R. C., Wang, X. J., and Liu, Y., Phys. Rev. Lett. 74, 546-549 (1995).

3. van Steenbergen, A., Gallardo, J., Sandweiss, J., Fang, J.-M., Babzien, M., Qiu, X., Skaritka, J., and Wang, X.J., Phys. Rev. Lett. 77, 2690 (1996).

4. Liu, Y., Cline, D. B., Wang, X. J., Babzien, M., Fang, J. M., Kusche, K. P., 1997 Particle Accelerator Conference, May 12-16, 1997, Vancouver, B.C., Canada, Paper 3'C3.

5. See Staged Electron Laser Acceleration (STELLA) Technical Proposal submitted to U.S. Department of Energy by STI Optronics, Inc., June 27, 1998.

6. Fontana, J. R. and Pantell, R. H., J. Appl Phys. 54, 4285-4288 (1983).

7. Romea, R. D. and Kimura, W. D., Phys. Rev. D 42, 1807-1818 (1990).

8. Steinhauer, L. C., and Kimura, W. D., "Space Charge Compensation in Laser Particle Accelerators," in these Proceedings of the 8th Workshop on Advanced Accelerator Concepts, Jul. 5-11, 1998, Baltimore, MD.

9. Kusche, K. P., Ben-Zvi, I., Babizen, M., Campbell, L. P., Cline, D. B., Fiorito, R. B., Gallardo, J. C., Gottschalk, S. C., He, P., Kimura, W. D., Liu, Y., Pantell, R. H., Pogorelsky, I. V., Quimby, D. C., Robinson, K. E., Rule, D. W., Sandweiss, J., Skaritka, J., Steinhauer, L. C., van Steenbergen, A., and Yakimenko, V., "STELLA Experiment - Hardware Issues," in these Proceedings of the 8th Workshop on Advanced Accelerator Concepts, Jul. 5-11, 1998, Baltimore, MD. 